\title{
Editorial
}

\section{Developmental Biology - Expanding the Horizon}

\author{
Andy Wessels \\ Department of Regenerative Medicine and Cell Biology, Cardiovascular Developmental Biology \\ Center, Medical University of South Carolina, 173 Ashley Avenue, Charleston, South Carolina 29425, \\ USA; E-Mail: wesselsa@musc.edu; Tel.: +1-843-792-8183 Fax: +1-843-792-0664
}

Received: 12 September 2012 / Accepted: 13 September 2012 / Published: 25 September 2012

Developmental biology is arguably the most exciting field of study within the biological sciences. To elucidate how complex organisms develop from a single cell into a complex organism is a quest that has captured the minds of many great scientists. The field of developmental biology comprises a wide variety of topics and uses a range of many different model systems. In the past, research in the field mainly focused on increasing our basic understanding of the elementary, yet complex, mechanisms that underlie the growth and development of animals and plants. As it has become increasingly clear that many of the concepts and paradigms developed within the context of developmental biological studies also apply to other conditions in life (e.g., cancer, wound healing, regeneration), the scope of developmental biology has even widened more and in many cases overlaps with other disciplines including genetics, cancer research, and regenerative medicine.

The primary goal of The Journal of Developmental Biology is to provide the scientific community with a platform to publish their scientific studies on the development of multicellular organisms at the molecular, cellular, tissue, organ, and whole organism levels in a speedy and efficient way. We also welcome papers in related fields, such as cancer and regenerative medicine, that have a clear developmental biological component. All papers submitted to The Journal of Developmental Biology will undergo a fast, yet thorough and rigorous, peer-review process to assess whether the work is of high quality. In this review process, strict ethical policies and standards will be applied. Reviewers will be specifically asked to focus on the quality of the conducted research, rather than on the impact or significance of the work in the field. After all, weighing the impact of a particular study is often difficult to determine (and can be very subjective) and what may seem to be a low-impact paper at one point may be considered a seminal paper in the future. We believe that if a study has been accurately conducted, if the methodology is sound and well described, and if the results are of quality, exciting and clearly reported, than that study is worthy of publication. While it often takes months before an 
accepted paper is published in other, more traditional, journals, the "open access" format of The Journal of Developmental Biology ensures fast dissemination of results to a large scientific audience.

While a "new arrival" in the field of journals that focus on developmental biology, we hope that The Journal of Developmental Biology will soon find its own niche. By including papers on "applied" or "translational" developmental biology we offer something that may be difficult to find in the competition. In an effort to facilitate this process, we plan to publish in the coming year a series of special issues, edited by experts in the respective disciplines, and an additional 20 original research papers. We strongly believe that with your help The Journal of Developmental Biology will quickly become a favorite venue for those that seek publication of their quality research without unnecessary delay.

(C) 2012 by the authors; licensee MDPI, Basel, Switzerland. This article is an open access article distributed under the terms and conditions of the Creative Commons Attribution license (http://creativecommons.org/licenses/by/3.0/). 